\title{
Performance Analysis of MANET Protocols for DTN
}

\author{
Madhusudan G \\ Asst.Professor, Department of CS\&E,SJCE,Mysore-570006 \\ Kumar TNR \\ Asst.Professor,MSRIT,Bangaluru
}

\begin{abstract}
Communication device nodes operation greatly depends on energy constraints such as battery power. The existing Bundle Protocol mainly concentrates on message transmission reliability. There is need for energy efficient routing in Delay Tolerant Network (DTN). Energy efficiency can be increased by allowing dead nodes for routing purpose but the dead nodes induces delay in transmission. The Routing Protocols like Dynamic Source Routing (DSR), Ad-hoc On-Demand Distance Vector (AODV)and Destination-Sequenced Distance-Vector(DSDV) Routing protocols can minimize the delay in transmission and enhance the throughput of the system.
\end{abstract}

\section{INTRODUCTION}

A wireless Network (WN) consists of spatially distributed autonomous devices, and a basestation to cooperatively monitor physical or environmental conditions, such as temperature, sound, vibration, pressure, motion and pollution, at different locations[6]. The nodes can be networked to gather data and each Node performs data sending activities, and Routing the data to the base station or a controller. There are two types of routing in adhoc network namely Proactive routing protocols [1-3] are based on either link-state [4] or distancevector [5] routing

schemes.

Characteristics of WN

WNs are characterized by the following Limited power they can harvest or store, Ability to cope with node failures, Heterogeneity of nodes, Large scale of deployment,Mobility of nodes, Communication failures and Dynamic network topology[7].

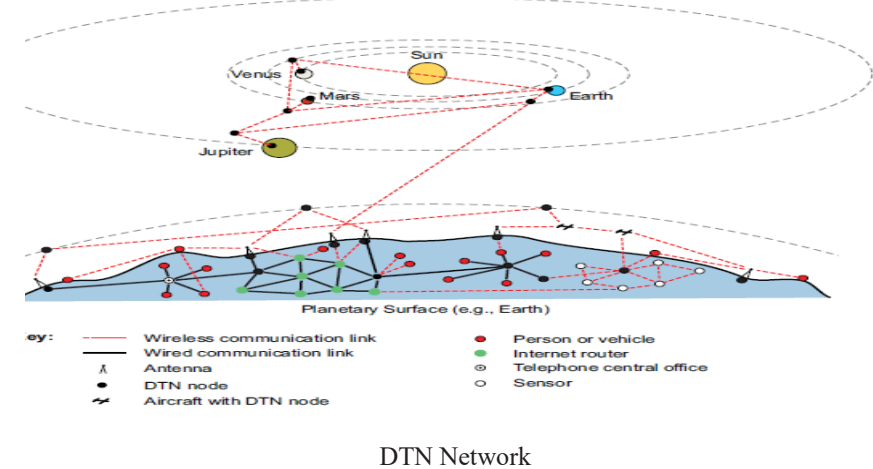

A DTN is a network of smaller networks. It is an overlay on top of special-purpose networks, including the Internet[8]. DTNs were originally developed for interplanetary use, where the speed of light can seem slow and delay-tolerance is the greatest need. DTNs can accommodate many kinds of wireless technologies, including radiofrequency (RF), ultra-wideband(UWB), free-space optical, and acoustic (sonar or ultrasonic) technologies. The DTN architecture system follows store-and-forward message switching by overlaying a new transmission protocol called the Bundle protocol[9][10].The Bundle protocol ties together the lower protocols so that application programs can communicate across the same or different sets of lower-lower protocols under conditions that involve long network delays or disruptions. The Bundle-protocol agent stores and forwards entire bundles (or bundle fragments) between nodes. A single Bundle protocol is used throughout a DTN. By contrast, the lower-level protocols below the Bundle protocol are chosen to suit the characteristics of each communication environment.

Bundle Encapsulation:The Bundles consist of three things: A bundle header consisting of one or more DTN blocks inserted by the bundle-protocol agent, A source-application's user data, including control information 
provided by the source application for the destination application that describes how to process, store, dispose of, and otherwise handle the user data. An optional bundle trailer, consisting of zero or more DTN blocks, inserted by the bundle-protocol agent like application user data, bundles can be arbitrarily long.

\section{II. $\quad$ ALGORITHM OF THE STATIC SCENARIO APPLICATION SYSTEM}

1. Start.

2. Initialization of nodes and deployment of the nodes to their respective positions.

3. Among the initialized nodes the central node which receives the information from all the clusters becomes the base station and this base station is an independent node and it does not belong to any cluster.

4. All the nodes that come under certain area forms a group of nodes called Cluster.

5. Among the nodes of a cluster one node will be selected as the Cluster Head for that cluster. Similarly Cluster heads for all the other clusters are selected.

6. Nodes that don't have sufficient energy will be declared as the dead nodes and dead nodes go to sleep state.

7. Once the Cluster Head is selected all the nodes other than cluster head and dead nodes transmit data to the respective Cluster Heads.

8. If (Sensors of the Base Station is within the range of dead node)

\{

Cluster Head activates the dead node for a short duration and sends the data to the Base Station through dead node.

\}

Else

\{

Cluster head searches for other optimum paths for routing.

\}

9. Dead node returns to its sleep state again.

10. End.

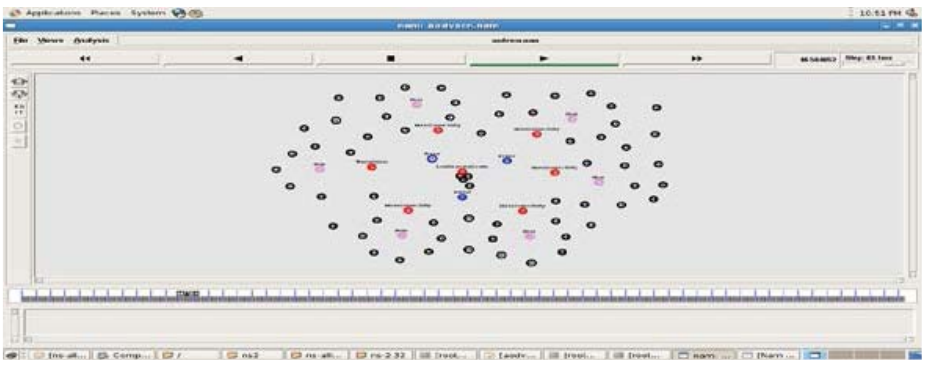

Implementation of Static Scenario of the system

Average energy v/s Simulation Time

From the Average Residual Energy v/s SimulationTime

The energies of the Nodes are same initially. After 70 seconds energies of the nodes with respect to DSDV and AODV will be more followed by DSR. But the difference will be very less.

At 140 seconds, nodes with respect to AODV will have more energy followed by DSDV and DSR. As Simulation time increases Residual energy decreases for all nodes. At 210 and 270 seconds energy of nodes decreases in the same manner for all protocols.

Delay v/s Simulation Time graph: We have taken the average delay for each 70s. From the Delay v/s Simulation Time bar graph,

After 70 seconds delay in transmission of data for AODV is less with respect to DSDV followed by DSR. But the difference will be very less.

At 140, 210 and 270 seconds delay in transmission of data follows the same order as that of 70 seconds with increase in delay.

Packet Delivery Ratio v/s Simulation Time graph

We have taken the Packet Delivery Ratio for each 70s along y-axis and time along x-axis. The graph is plotted using Microsoft Excel to look simpler and enhance the visibility.

From the Packet Delivery Ratio (PDR) v/s Simulation Time bar graph,

The PDR is very high almost $100 \%$ at 70 seconds. 
At 140 seconds, PDR with respect to AODV almost remains same but the PDR of DSDV and DSR decreases considerably.

At 210 seconds, PDR of AODV decreases but PDR of DSDV remains same as it was at 140 seconds. PDR of DSR decreases considerably comparing to AODV and DSDV.

At 250 seconds, PDR with respect to AODV almost remains same as it was at 210 seconds but the PDR of DSDV and DSR decreases.

\section{MOBILITY/DYNAMIC SCENARIO OF THE APPLICATION SYSTEM}

The flow chart for rescue operation after catastrophic disaster is shown in Figure below.

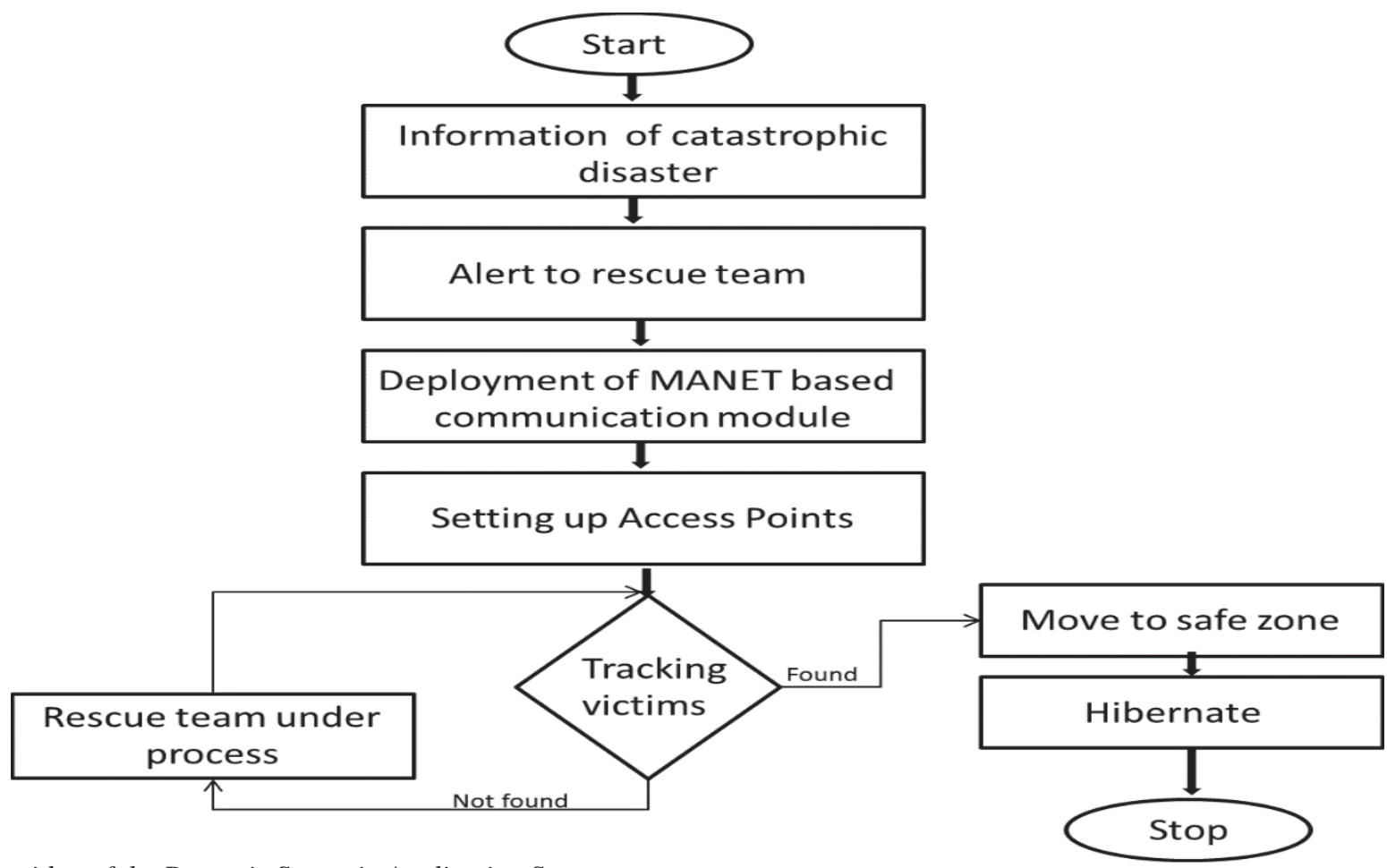

Algorithm of the Dynamic Scenario Application System:

1.Initially the information of the disaster and the area under investigation is acquired. The nearby activated base station transceiver systems (BTS) is located and a wired access point is deployed to provide connectivity.

2. The rescue team is alerted for the disaster response.MANET based emergency communication network is deployed. The access points are air dropped around the disaster stuck area with the help of rescue team choppers. Due to its unique structure it lands vertically or floats over water and is ready for network communication instantly.

3. The mobile devices used by the victims will automatically get latched to the nearby access point network. The victim in the disaster struck area will come under any of the access points, and by this the exact location of the victim is tracked. The condition around the victim is also predicted and all these information is sent to the rescue brigades.

4. The rescue operations are undertaken and the victim is moved to the safe zone. The information gathered from the rescue brigade's headgear helps the base station to investigate the complete post-disaster scenario.

5. The rescue teams are allowed to communicate amongst each other when it is need to direct themselves to a particular remote location.

6. Whenever the rescue brigades fail to find the victims at a location, an alert is sent to the base station to resend the information about the victim's location, and the rescue operation resumes.

7. Whenever the rescue brigades fail to find the victims at a location, an alert is sent to the base station to resend the information about the victim's location, and the rescue operation resumes. 


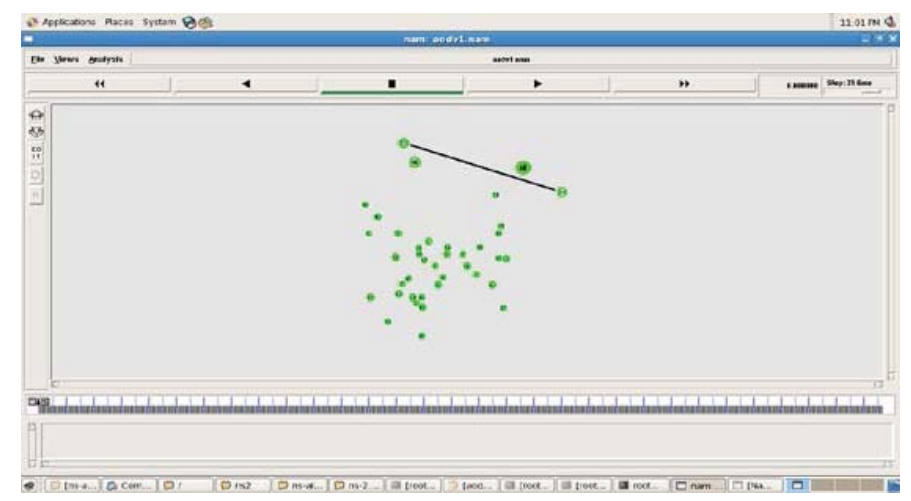

Mobility Scenario:

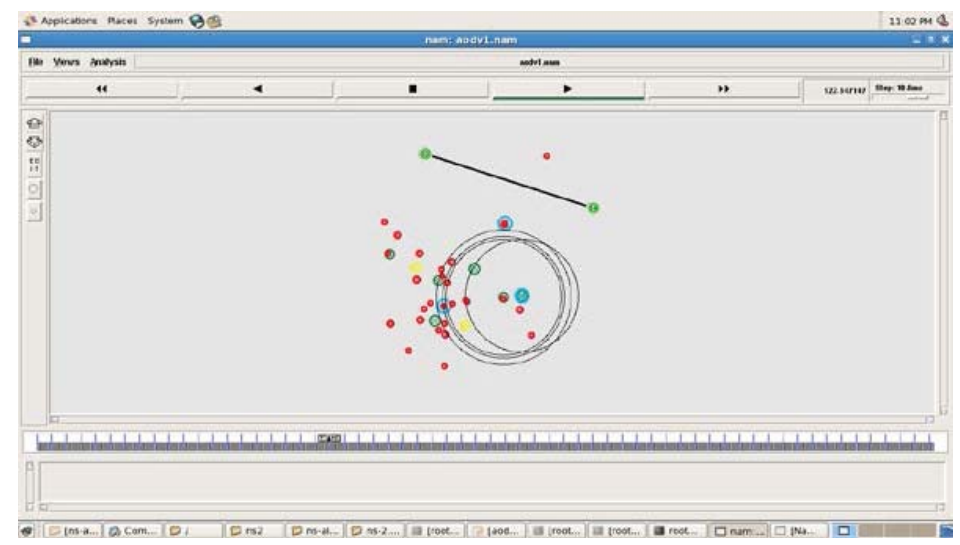

Dynamic or Mobility Scenario:

Average energy v/s Simulation Time graph:We have taken the average energy for each 70s along y-axis and time along X-axis. From the Average Residual Energy v/s Simulation Time bar graph,

The energies of the Nodes are same initially. After 70 seconds energies of the nodes with respect to DSDV will be more followed by DSR and AODV. But the difference is very less.

At 140 seconds, nodes with respect to AODV will have more energy followed by DSDV and DSR. As Simulation time increases Residual energy decreases for all nodes. At 210 and 270 seconds energy of nodes decreases in the same manner for all protocols.

Delay v/s Simulation Time graph: We have taken the average delay for each 70s along y-axis and Simulation time along x-axis. From the Delay v/s Simulation Time bar graph,

After 70 seconds delay in transmission of data for DSDV is less with respect to AODV and DSR. But the difference will be very less.

At 140 seconds delay in transmission of data is more for DSR but less delay for DSDV followed by AODV. At 210 and 270 seconds delay in transmission of data follows the same order as that of 140 seconds with decrease in delay.

Packet Delivery Ratio v/s Simulation Time Bar graph:We have taken the Packet Delivery Ratio for each 70s From the Packet Delivery Ratio (PDR) v/s Simulation Time graph,

The PDR is low at 70 seconds. At 140 seconds, PDR of all the protocols increases as number of nodes and network traffic decreases. At 210 seconds, order of PDR is same as that of 140 seconds.

At 250 seconds, PDR with respect to AODV is the highest followed by PDR of DSR and DSR.

\section{CONCLUSION \& FUTURE WORK}

In our work, we identified some of the important design issues of routing protocols for sensor networks (DTN) and also compared and contrasted the existing routing protocols. As our study reveals, it is not possible to design a routing algorithm which will have good performance under all scenarios and for all applications. Although many routing protocols have been proposed, many issues still remain to be addressed. 
We studied energy consumption of three adhoc routing protocols (AODV, DSR and DSDV) with a simple traffic model in which a few nodes send data over a multi-hop path. With this energy model we found that ondemand protocols such as AODV and DSR consume much less energy than a priori protocol such as DSDV. This makes sense since a priori protocol is constantly expending energy pre-computing routes, even though there is no traffic passing on these routes.

In our work, we have presented proposal for energy efficient emergency communication module to manage disasters further investigation on performance analysis of this schema in different network scenarios can be taken as extended network. We have investigated post-disaster communication from the viewpoint of time elapsed and classified communication based on purpose, sources, destinations and contents of communications for each transmitter and receiver. Theresults gained from simulations show that performance of DSR is better than AODV and DSDV in residual energy plots.Finally, experimentation is needed to validate these results with physical hardware in actual scenarios. Therefore, more research is needed to combine and integrate some of the protocols presented in this work to keep the network functioning for a longer duration.

\section{REFERENCES}

[1] C.E. Perkins, P. Bhagwat, Highly dynamic destination-sequenced distance-vector routing (DSDV) for mobile com-puters, in: Proceedings of the ACM SIGCOMMÕ94, London, UK, September 1994, pp. 234-244.

[2] G. Pei, M. Gerla, T.-W. Chen, Fisheye state routing: a routing scheme for ad hoc wireless networks, in: Proceedings of the IEEE ICC 2000, New Orleans, LA, June 2000, pp. 70-74.

[3] J.J.Garcia-Luna-Aceves, M. Spohn, Source-tree routing in wireless networks, in: Proceedings of the IEEE ICNP 99, Toronto, Canada, November 1999, pp. 273-282.

[4] G.S. Malkin, M.E. Steenstrup, Distance-vector routing, in: M. Steenstrup (Ed.), Routing in Communications Networks, Prentice Hall, Englewood Cliffs, NJ, 1995, pp. 83-98.

[5] J. Moy, Link-state routing, in: M. Steenstrup (Ed.), Routing in Communications Networks, Prentice Hall Inc., Engle-wood Cliffs, NJ, 1995, pp. 135-157.

[6] M. Rizwan Pasha, Mahaveer Jayakkanavar (2012), MANET Based Dynamic Power Consious Disaster Management System. National Conference at SJBIT, Bangalore.

[7] Kapang Lego, Pranav Kumar Singh, Dipankar Sutradhar (2006), Comparative Study of Adhoc Routing Protocol AODV, DSR and DSDV in Mobile Adhoc NETwork, Issue of IJCSE Vol.1-No.4364-371.

[8] Suvarna Patil, Geetha R. Chillerge (2014). “Delay Tolerant Networks - Survey Paper”. Suvarna Patil at Int. Journal of Engineering Research and Applications ISSN: 2248- 9622, Vol. 4, Issue 2(Version 2), February 2014.

[9] JorgOtt, Mikko Juhani Pitkanen (2007). “DTN-based Content Storage and Retrieval”.Helsinki University of Technology (TKK).

[10] Aruna Balasubramanian, Brian Neil Levine and Arun Venkataramani (2007). "DTN Routing as acResourceAllocation Problem”.SIGCOMM'07,cAugust 27-31, 2007,Kyoto, Japan. 